EPJ Web of Conferences 47, 01002 (2013)

DOI: $10.1051 /$ epjconf/20134701002

(C) Owned by the authors, published by EDP Sciences, 2013

\title{
Hot Jupiters around M dwarfs
}

\section{A sensitivity analysis of the WFCAM Transit Survey}

Gábor Kovács ${ }^{1, a}$, S. Hodgkin ${ }^{1}$, B. Sipőcz² ${ }^{2}$ D. Pinfield², D. Barrado ${ }^{3}$, J. Birkby ${ }^{4}$, M. Cappetta ${ }^{5}$, P. Cruz ${ }^{3}$, J. Koppenhoefer ${ }^{5}$, E. Martín' ${ }^{6}$, F. Murgas ${ }^{6}$, B. Nefs ${ }^{4}$, R. Saglia ${ }^{5}$ and J. Zendejas ${ }^{5}$

1 Institute of Astronomy, University of Cambridge, Cambridge CB3 OHA, UK

${ }^{2}$ Centre for Astrophysics Research, University of Hertfordshire, College Lane, Hatfield

AL10 9AB, UK

${ }^{3}$ Centro de Astrobiología, Inst. Nacional de Técnica Aeroespacial, 28850 Torrejón de Ardoz, Madrid, Spain

${ }^{4}$ Leiden Observatory, Universiteit Leiden, Niels Bohrweg 2, 2333 CA Leiden,

The Netherlands

${ }^{5}$ Max-Planck-Institut für extraterrestrische Physik, Giessenbachstraße, 85748 Garching,

Germany

${ }^{6}$ Instituto de Astrofísica de Canarias, C/ Vía Láctea s/n, 38205 La Laguna (Tenerife), Spain

\begin{abstract}
The WFCAM Transit Survey (WTS) is a near-infrared transit survey running on the United Kingdom Infrared Telescope (UKIRT). We conduct Monte Carlo transit injection and detection simulations for short period ( $<10$ day) Jupiter-sized planets to characterize the sensitivity of the survey. We investigate the recovery rate as a function of period and magnitude in 2 hypothetical star-planet cases: M0-2 + hot Jupiter, M2-4 + hot Jupiter. We find that the WTS lightcurves are very sensitive to the presence of Jupitersized short-period transiting planets around $\mathrm{M}$ dwarfs. The non-detection of a hot-Jupiter around an $\mathrm{M}$ dwarf by the WFCAM Transit Survey allows us to place a firm upper limit of 1.9 per cent (at 95 per cent confidence) on the planet occurrence rate.
\end{abstract}

\section{INTRODUCTION}

M dwarfs are the most numerous stars in our Galaxy, and until recently have remained relatively unexplored as exoplanet hosts. Around solar-type stars, planets in short period orbits (days) are extremely hot (above $1000 \mathrm{~K}$ ). If $\mathrm{M}$ dwarfs harbour planets in equally close-in orbits, they will probably be more interesting due to their moderate equilibrium temperatures. $\mathrm{M}$ dwarfs are also important for testing planet formation theories. In the core accretion paradigm, dust particles in the protoplanetary disk coagulate and form solid cores. The cores then begin to accumulate gas from the disk which can speed up significantly when cores reach several Earth masses. It was first shown by [1] that gas giants cannot form easily around low mass stars this way. Revised models [2-4] allow gas giant formation around low mass stars but the predicted frequency of giants systematically decreases towards lower primary masses.

\footnotetext{
ae-mail: gkovacs@ast.cam.ac.uk
}

This is an Open Access article distributed under the terms of the Creative Commons Attribution License 2.0, which permits unrestricted use, distribution, and reproduction in any medium, provided the original work is properly cited. 
Even if it is hard to form giant planets via core accretion around the lowest mass stars, gravitational instability models can produce gas giants on a very short timescale $\left(\sim 10^{3} \mathrm{yr}\right)$. They predict that gas giants can form around low mass primaries as efficiently as around more massive ones assuming that the protoplanetary disk is sufficiently massive to become unstable [5].

Having these scientific motivations, the WFCAM transit survey (WTS) was designed to target a high number of M dwarfs. It is a bad weather fallback programme on the United Kingdom Infrared Telescope (UKIRT) operating since 2007. The WTS has four seasonally varied fields. The fallback nature of the project provides varying time coverage usually taking 3-4 observations at the beginning of the nights but giving a long overall baseline (years). In this short study, we use the most complete $19 \mathrm{hr}$ field with 950 epochs to determine the survey's sensitivity for short period giant transiting planets. For a more in depth description of the WTS sensitivity we refer to [6].

\section{SURVEY SENSITIVITY SIMULATION}

In general, the probability of detecting a transiting system in the survey can be written as [7]:

$$
P_{\mathrm{det}}=\iint P_{r} P_{T} p\left(R_{p}, T\right) d R_{p} d T
$$

where $P_{r}$ is the average recovery ratio, i.e. the average (conditional) probability of recovering a transit from a lightcurve if the lightcurve belongs to a transiting system, $P_{T}$ is the geometric probability of having a transit in a randomly oriented planetary system, and $p\left(R_{p}, T\right)$ is the joint probability density function of $R_{p}$ and $T$ for planetary systems. We determine these terms in Eq. 1 separately for two starplanet scenarios: earlier (M0-2) and later (M2-4) M dwarfs + Jupiter sized planet. We use conservative assumptions for the stellar parameters: stellar radii are overestimated, using the maximum values in each spectral bin (i.e at M0 and M2), and planet radii are (under)estimated assuming solar system radii even for hot planets.

To determine $P_{r}$, we use a Monte Carlo approach and simulate large numbers of transiting exoplanet systems to determine the recovery ratios for the two scenarios under investigation. In each iteration, a transiting planetary system is created with parameters randomly drawn from fixed prior distributions. A simulated transit signal is then added to the randomly selected quiet lightcurve (from a pre-selected sample of 4700 real WTS lightcurves spanning J magnitudes of 11-17, and having originally a BLS $S_{\text {red }}<6$ detection in the survey). We then try to recover the artificial system using the BLS transit detection algorithm [8]. $P_{r}$ is estimated as the ratio between successful transit recoveries and the total number of iterations. We make a distinction between two different cases. In the threshold case we consider the signal successfully recovered if the detection passes the same signal to red noise level as used for WTS candidate selection $\left(S_{\text {red }}>6\right)$ in the survey. In the periodmatch case, we additionally require that the recovered period value matches the simulated one.

Sensitivity results are shown in figure 1 left panel. The WTS sensitivity for Jupiter sized planets has a maximum around $\mathrm{J}=13.5$ and drops towards fainter $(\mathrm{J}>15-16)$ objects. This is in accordance with our expectations, we have higher noise levels towards fainter objects but there is occasional saturation at the bright end $(J<13)$. There is little dependence on stellar radius, appearing only for the fainter stars. The threshold curves are less affected by simulated period than the periodmatch recovery rate. In the shortest period window the threshold and periodmatch values are practically the same. In the longest period panels the threshold ratio is about twice that of the periodmatch ratio showing that while the signal-to-noise detection statistic can recover signals, many of these systems may be missed due to poor initial period guesses.

Overall detection probabilities can now be obtained by integrating eq. 1 over a selected prior for the period distribution. We use a power law prior with an exponential cut on the short period end from [9], as derived from Kepler data for Jupiters around solar type stars. 


\section{Hot Planets and Cool Stars}
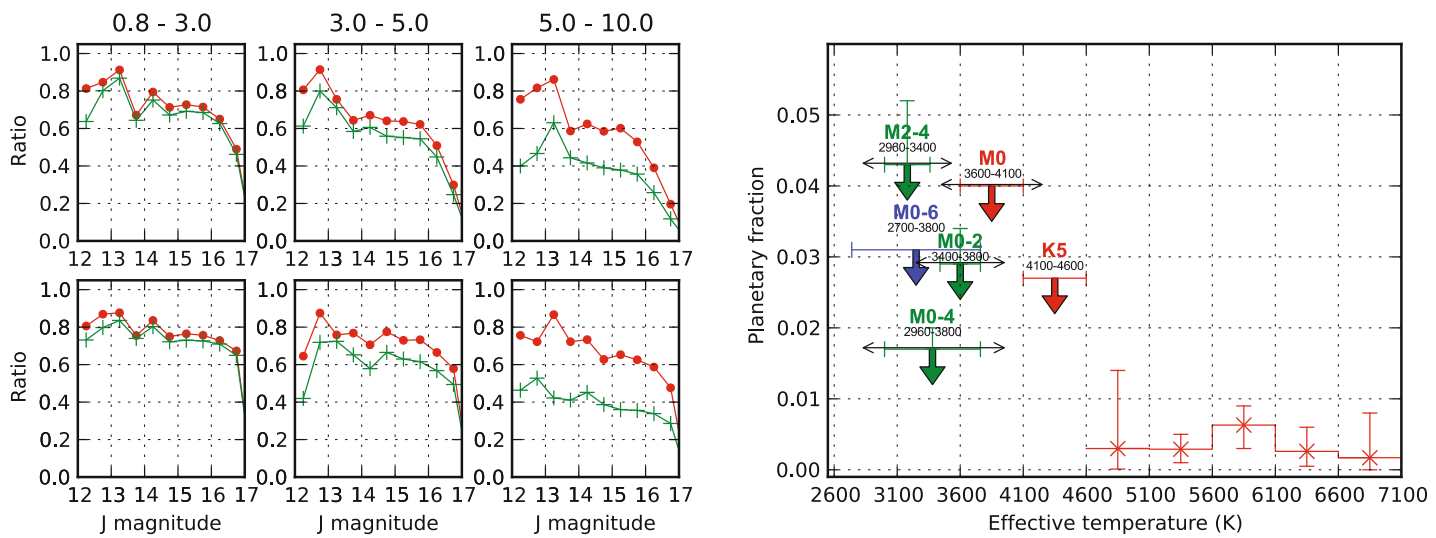

Figure 1. Recovery rates of injected planetary signals into light curves of $M$ dwarfs in the WTS (left) and 95\% confidence planetary occurrence rates of short period giants (right, [6]). Recovery rates are shown for the threshold (red) and periodmatch (green) cases around M2-4 (upper row) and M0-2 (bottom row) stars. In the right panel short period giant planet occurrence rates around $\mathrm{M}$ dwarfs (green, M0-2, M2-4, M0-4) in the WTS are compared to fractions based on [9] (red, for M and earlier dwarfs) and from the [10] RV study (blue, M0-6).

\section{OCCURRENCE RATES OF HOT JUPITERS}

A summary of occurrence rates of hot giant planets ( $T<10$ days) around FGK type dwarfs is presented in [11] and references therein. RV studies determined a rate of $0.9-1.5 \%$ [11-14], while transit studies have a systematically lower rate (roughly half of this value) at $0.3-0.5 \%[9,15]$.

For M dwarfs, recent RV studies support the paucity of giant planets [13, 16-18] though there are confirmed detections both with short- and long-period orbits. [16] found 3 Jovian planets (with orbital periods of years) in a sample of $169 \mathrm{~K}$ and $\mathrm{M}$ dwarfs, in the California and Carnegie Planet Search data. The planetary occurrence rate for stars with $\mathrm{M}<0.7 \mathrm{M}_{\odot}$ in their survey is $1.8 \%$, which is significantly lower than the rate found around more massive hosts (4.2\% for Solar-mass stars, $8.9 \%$ around higher mass subgiants).

Focusing on giant planets with short orbital periods around $\mathrm{M}$ dwarfs, then as of writing, there is only one confirmed detection. The Kepler Mission [19], includes a sample of 1086 low-mass targets (in Q2) $[9,20]$ and one confirmed hot Jupiter ( $\mathrm{P}=2.45$ days) around an early M dwarf host (KOI-254) [21]. Unfortunately, this object was not included in the statistical analysis of the [9] study.

Using our integrated sensitivity values for Jupiter sized planets, and considering the size of the M dwarf sample of the WTS, the null result for Jupiters around M dwarfs in the survey can be turned into statistical upper limits. With 95\% confidence, we can conclude that fewer than 4.3-5.2\% of M2-M4 dwarfs host hot Jupiters (periods $<10$ days). Constraints are even stronger for earlier spectral types, and fewer than 2.9-3.4\% of M0-M2 dwarfs host hot Jupiters. Combining the two bins, this means an upper limit of $1.7-2.0 \%$ for the whole M0-4 spectral range (figure 1, right panel, green markers).

For comparison, we conducted a brief re-calculation of the [9] results based on the Kepler Q2 data release. These occurrence rates for the same period interval are shown by red markers in the right panel of figure 1. " $\downarrow$ " mark upper limits in case of null detections, while " $x$ " markers are for non-null fractions. The upper limit (rescaled to 95\% confidence) is also shown from the [10] study.

We can conclude that the WTS provides a more rigorous upper limit than the [9] Kepler study, thanks to the larger size of our sample of early M dwarfs (2844 M0-2 dwarfs in the WTS compared to 1086 M0 stars in the Kepler sample under consideration). The limits for both M0-2 and M2-4 bins are consistent with the [10] results. Considering the M dwarf sample of the $19 \mathrm{hr}$ field in the WTS as one unified bin, as of writing, the WTS can provide the strictest upper limit on short period giant planets 
around $\mathrm{M}$ dwarfs based on null detections. Comparing these upper limits to the measured hot Jupiter fraction around more massive host stars, we find that they are consistent, i.e. we cannot rule out similar planet formation scenarios around at least the earlier M dwarfs (M0-4) at the moment.

\section{References}

[1] G. Laughlin, P. Bodenheimer, F.C. Adams, ApJ, 612, L73 (2004), arXiv : astro-ph/0407309

[2] S. Ida, D.N.C. Lin, ApJ, 626, 1045 (2005), arXiv : astro-ph/0502566

[3] S. Ida, D.N.C. Lin, ApJ, 685, 584 (2008)

[4] G.M. Kennedy, S.J. Kenyon, ApJ, 673, 502 (2008), 0710.1065

[5] A.P. Boss, ApJ, 643, 501 (2006), arXiv: astro-ph/0601486

[6] G. Kovács et al., submitted to MNRAS, (2013)

[7] J.D. Hartman, B.S. Gaudi, M.J. Holman, B.A. McLeod, K.Z. Stanek, J.A. Barranco, M.H. Pinsonneault, S. Meibom, J.S. Kalirai, ApJ, 695, 336 (2009), 0809.3807

[8] S. Aigrain, M. Irwin, MNRAS, 350, 331 (2004), arXiv : astro-ph/0401393

[9] A.W. Howard, G.W. Marcy, S.T. Bryson, J.M. Jenkins, J.F. Rowe, N.M. Batalha, W.J. Borucki, D.G. Koch, E.W. Dunham, T.N. Gautier, III et al., ApJSupp, 201, 15 (2012), 1103. 2541

[10] X. Bonfils, X. Delfosse, S. Udry, T. Forveille, M. Mayor, C. Perrier, F. Bouchy, M. Gillon, C. Lovis, F. Pepe et al., ArXiv e-prints (2011), 1111.5019

[11] J.T. Wright, G.W. Marcy, A.W. Howard, J.A. Johnson, T.D. Morton, D.A. Fischer, ApJ, 753, 160 (2012), 1205. 2273

[12] G. Marcy, R.P. Butler, D. Fischer, S. Vogt, J.T. Wright, C.G. Tinney, H.R.A. Jones, Progress of Theoretical Physics Supplement 158, 24 (2005), arXiv: astro-ph/0505003

[13] A. Cumming, R.P. Butler, G.W. Marcy, S.S. Vogt, J.T. Wright, D.A. Fischer, PASP, 120, 531 (2008), 0803.3357

[14] M. Mayor, M. Marmier, C. Lovis, S. Udry, D. Ségransan, F. Pepe, W. Benz, J.. Bertaux, F. Bouchy, X. Dumusque et al., ArXiv e-prints (2011), 1109.2497

[15] A. Gould, S. Dorsher, B.S. Gaudi, A. Udalski, AcA, 56, 1 (2006), arXiv: astro-ph/0601001

[16] J.A. Johnson, R.P. Butler, G.W. Marcy, D.A. Fischer, S.S. Vogt, J.T. Wright, K.M.G. Peek, ApJ, 670, 833 (2007), 0707.2409

[17] J.A. Johnson, K.M. Aller, A.W. Howard, J.R. Crepp, PASP, 122, 905 (2010), 1005. 3084

[18] F. Rodler, R. Deshpande, M.R. Zapatero Osorio, E.L. Martín, M.M. Montgomery, C. Del Burgo, O.L. Creevey, A\&A, 538, A141 (2012), 1112.1382

[19] W.J. Borucki, D.G. Koch, E.W. Dunham, J.M. Jenkins, The Kepler Mission: A Mission To Detennine The Frequency Of Inner Planets Near The Habitable Zone For A Wide Range Of Stars, in Planets Beyond the Solar System and the Next Generation of Space Missions, edited by D. Soderblom (1997), Vol. 119 of Astronomical Society of the Pacific Conference Series, p. 153

[20] W.J. Borucki, D.G. Koch, G. Basri, N. Batalha, T.M. Brown, S.T. Bryson, D. Caldwell, J. Christensen-Dalsgaard, W.D. Cochran, E. DeVore et al., ArXiv e-prints (2011), 1102.0541

[21] J.A. Johnson, J.Z. Gazak, K. Apps, P.S. Muirhead, J.R. Crepp, I.J.M. Crossfield, T. Boyajian, K. von Braun, B. Rojas-Ayala, A.W. Howard et al., AJ, 143, 111 (2012), 1112.0017 